\title{
A Two-Phase SPH Model for Dynamic Contact Angles Including Fluid-Solid Interactions at the Contact Line
}

\author{
P. Kunz ${ }^{1}$ (D) S. M. Hassanizadeh ${ }^{2}$ - U. Nieken ${ }^{1}$
}

Received: 17 January 2017 / Accepted: 10 January 2018

(C) Springer Science+Business Media B.V., part of Springer Nature 2018

\begin{abstract}
The description of wetting phenomena on the continuum scale is a challenging problem, since intermolecular interactions, like van der Waals forces between liquid and solid, alter the flow field at the contact line. Recently, these effects were included in the smoothed particle hydrodynamics method by introducing a contact line force (CLF) on the continuum scale. This physically based contact line force model is employed here to simulate two-phase flow in a wide range of wetting dynamics parametrized by capillary number. In particular, dynamic contact angles at various capillary number values are calculated by CLF model and compared to measured values. We find that there is significant disagreement between simulated and measured results, specially at low wetting speeds. It is indeed expected that most of the driving force is dissipated to overcome strong liquid-solid interactions, which are not adequately accounted for in the existing CLF model. Therefore, we have extended that model to account for stick-slip (SSL) behavior of the contact line caused by solid-fluid interactions. The new SSL model results in dynamic contact angle values that are in good agreement with experimental data for the full range of wetting dynamics.
\end{abstract}

Keywords Dynamic contact angle · Contact line force · Two-phase flow · Stick-slip behavior $\cdot$ Smoothed particle hydrodynamics $(\mathrm{SPH})$

\section{List of symbols}

$\begin{array}{ll}A & \text { Arbitrary field variable } \\ B & \text { Transition function for continuous surface force at solid boundary } \\ c & \text { Color function }\end{array}$

P. Kunz

philip.kunz@icvt.uni-stuttgart.de

1 Institute of Chemical Process Engineering, University of Stuttgart, Böblinger Straße 78, 70199 Stuttgart, Germany

2 Department of Earth Sciences, Faculty of Geosciences, Utrecht University, 3508 TA Utrecht, The Netherlands 


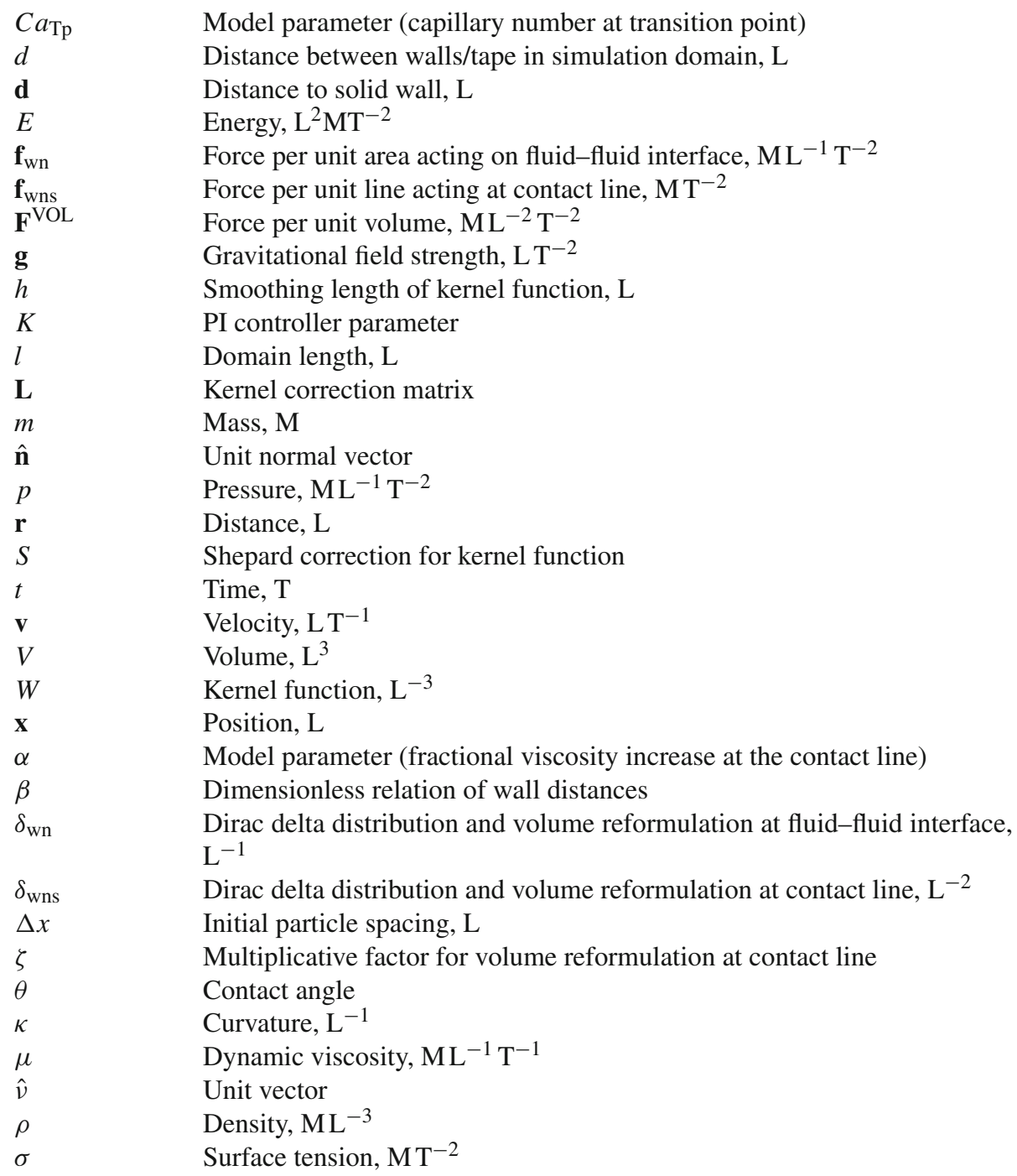

\section{Introduction}

Wetting phenomena are of great importance to numerous engineering applications like ink penetration into paper (Aslannejad et al. 2016), water transport in gas diffusion layers of a fuel cell (Hao and Cheng 2010) or bubble formation processes in a bubble column reactor (Huber et al. 2016a). Numerical modeling of these processes at the microscale is essential for understanding and predicting two-phase flow applications.

\subsection{Influence of Microscopic Surface Heterogeneity on Two-Phase Flow Dynamics}

In various numerical studies, reasonable agreements have been found between experiments and different pore-scale simulation methods regarding the general flow patterns [cf, pore 
(a)

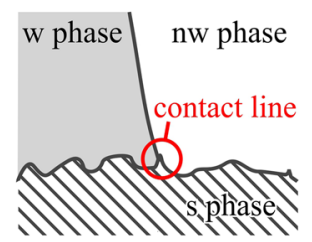

(b)

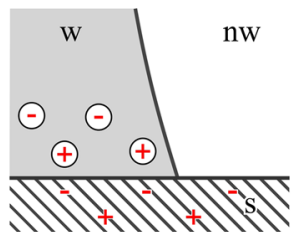

(c)

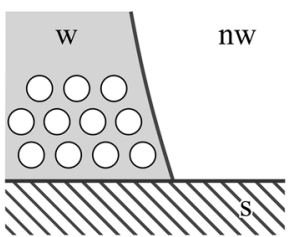

(d)

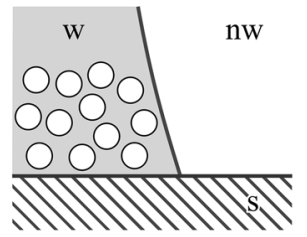

Fig. 1 Different microscopic effects that influence contact line movements, with "w" and "nw" denoting the wetting and non-wetting fluid phases, respectively. (a) Surface roughness. (b) Chemical heterogeneities induced by chemical bonding sites. Thermodynamic instability between ordered state (c) and disordered sliding state (d)

network modeling by Joekar et al. (2009); volume of fluid method by Ferrari et al. (2015) and smoothed particle hydrodynamics by Kunz et al. (2016b)]. In most simulations, usually the flow rate is given as the boundary condition. In the work of Kunz et al. (2016b), external pressure gradients were applied in the simulation and experiments. There, significantly larger disagreement was found between simulations and experiments for slower drainage processes. The capillary number, $C a=\left(\mu v_{\mathrm{cl}}\right) / \sigma_{\mathrm{wn}}$, during drainage experiments was less than $10^{-4}$, with $\mu, v_{\mathrm{cl}}$ and $\sigma_{\mathrm{wn}}$ being the liquid viscosity, contact line velocity, and fluid-fluid surface tension, respectively. We suppose that the drainage process happened in a stick-slip regime, where the contact line movement is impeded by microscale interactions between solid and the two fluid phases. Stick-slip behavior can be understood as irregular movement of the interface, with a non-constant, oscillating apparent contact angle.

Including the effects of fluid-solid interactions in a continuous CFD model in a general manner is a difficult task, since those interactions depend on local surface properties. For example, Johnson and Dettre (1964) studied effects of surface roughness (Fig. 1a) on the wettability of an idealized heterogeneous surface. Also, de Gennes (1985) showed how the receding contact angle varies with the degree of roughness. Later, de Gennes et al. (2003) determined the amount of energy necessary when a contact line is displaced across a single defect on an otherwise smooth surface. In this case, the fluid-fluid interface gets continually deformed until snap-off happens and the reversible energy, stored in the deformed interface, is lost as viscous dissipation. Recently, Wang et al. (2015) described how a contact line friction parameter, derived from the model of de Gennes et al. (2003), can be adjusted to fit wetting dynamics of a drop on a surface with defined roughness.

Pinning of the contact line can also be apparent on a flat but chemically heterogeneous surface (Extrand 2002), where discrete chemical bonding sites on the solid surface interact with the fluid molecules (Fig. 1b).

Yet another mechanism for pinning was proposed by Thompson and Robbins (1990) who claimed that stick-slip motion is rather caused by thermodynamic instability of the sliding state (Fig. 1c, d).

\subsection{Experimental Data Utilized for Quantitative Comparison with SPH Simulations}

The simulations performed in this work (see Sects. 3, 4.1) are compared with experiments where water or aqueous glycerol solutions wet untreated PET tape (Blake 1993, 2012; Blake and Shikhmurzaev 2002). In the experiment, a tape was vertically guided into a 2.5 -1 glass tank of rectangular shape. The contact angle was measured when the tape invaded the fluid in the 
Fig. 2 Experimental setup for measuring dynamic contact angles for a wide range of wetting speeds (Reproduced with permission from Blake and Shikhmurzaev 2002)

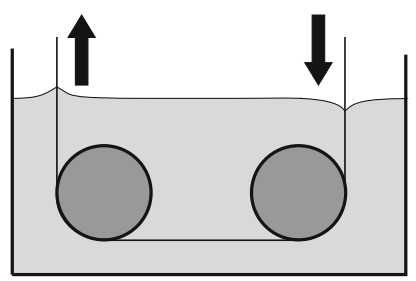

tank. In the glass tank, it passed two rollers and emerged out of the tank again (see Fig. 2). The setup was described in detail by Blake and Shikhmurzaev (2002). The advantage of using a tape was the very good observability on the dynamic contact angle during the wetting process. Blake (2012) found that when pure water was used two very different wetting modes existed. During fast wetting, the viscous dissipation was dominating, whereas at slow wetting most of the driving force was needed to overcome strong chemical bonds between the polar sites of PET and water molecules. Similarly, in earlier experiments, Blake and Shikhmurzaev (2002), who used aqueous glycerol, found that fluid-solid interactions become the dominant dissipation source for capillary numbers less than $10^{-2}$. The interactions with the wall caused a stick-slip behavior of the contact line. Such a behavior was already reported in experiments by Blake (1969). In the study of Blake (1969), glass capillaries were used and stick-slip behavior was found to appear for $\mathrm{Ca}<5 \times 10^{-5}$.

\subsection{Modeling of Contact Line Movement with Different CFD Methods}

At present, in microscale simulations of two-phase flow involving partially wetting fluids, a constant contact angle is usually assigned as boundary condition of the simulation domain (cf. Ferrari et al. 2015). There have been some efforts to include fluid-wall interactions in pore-scale multi-phase models in various simulation methods. Sheng and Zhou (1992) studied the dynamics of immiscible fluid displacement using a finite difference method and derived a slip boundary condition to capture dynamic contact angles. They found that an additional friction is necessary to match simulation results with experimental data of twophase flow at $C a<10^{-3}$. This additional friction was introduced by a velocity dependence of the microscopic contact angle on the capillary number. Francois and Shyy (2003) used the immersed boundary method to simulate the impact of a liquid drop on a flat surface surrounded by a gas and compared a static contact angle model with a dynamic one. It was shown that the model including the dynamic contact angle predicted a significantly reduced recoiling of the drop and better agreement with experimental results could be achieved. In their model, the dynamic contact angle varied linearly, between maximum advancing and minimum receding values, depending on the contact line velocity $v_{\mathrm{cl}}$. Chen et al. (2009) used the model of Francois and Shyy (2003) for the dynamic contact angle when simulating the shape and motion of elongated bubbles in capillary tubes (Taylor flow, see Angeli and Gavriilidis 2008) with the level set method of Sussman et al. (1998). It was shown that this method can cause unphysical behavior of the contact line movement. For example, it predicted that the contact line can move backwards even when the contact line velocity was positive or vice versa. Also Renardy et al. (2001) compared two methods of implementing a contact angle with the volume of fluid method (VOF). They found that the so- called three-phase method (Lafaurie et al. 1994) is free of the unphysical behavior of a backward moving contact line, since the contact line is driven by a physically based surface stress. Similar to the three-phase approach in the grid-based VOF method, moving contact lines were implemented in the Lagrangian 
smoothed particle hydrodynamics method (Hu and Adams 2006). The discretization in the work of $\mathrm{Hu}$ and Adams (2006) is mass conservative and showed qualitative agreement with molecular dynamic simulations of Thompson and Robbins (1989) without the need of special slip boundary conditions at the contact line. Unlike the formulation in $\mathrm{Hu}$ and Adams (2006), we make use of the continuum surface force model with the SPH discretization of Adami et al. (2010); hence, it does not conserve the total momentum of the system.

Wetting dynamics have been already studied with the SPH method by Tartakovsky and Meakin (2005). Unlike directly simulating surface tension, they applied pairwise fluid-fluid and fluid-solid particle-particle interactions to simulate three-phase contact dynamics. They found qualitatively good agreement with experimental results for the movement of a drop confined between parallel walls under the influence of gravity. Nevertheless, the formation of resolution-dependent films of the wetting fluid on the solid is still a unsolved issue for this type of surface tension model.

In our work, we use the incompressible SPH (ISPH) method based on the projection method introduced by Cummins and Rudman (1999). Compared with the widely used weakly compressible SPH (WCSPH) method (a review of this method is given by Monaghan (2005)), ISPH gives more accurate solutions of the pressure field and is therefore more suitable for a detailed analysis of the mobility of the contact line. Total mass conservation in the system is achieved using the ISPH algorithm of Hu and Adams (2007) to enforce both the zerodensity-variation condition and the velocity-divergence-free condition at each full time step. To increase accuracy, kernel corrections (Bonet and Lok 1999) are applied.

Recently, Huber et al. (2016b) introduced a contact line force (CLF) model, which uses a momentum balance for the three-phase contact line to calculate the unbalanced Young force, as proposed by de Gennes (1985), Brochard (1989) or Hassanizadeh and Gray (1993). This force is implemented by a volume reformulation in the vicinity of the contact line to transfer the force per line into a force per volume for direct applicability in the continuous NavierStokes equations. Since this model is a physically motivated approach on the continuum scale, no fitting parameters are necessary and the driving force calculated from dynamic contact angles leads to a dynamic evolution of the system, similar to the three-phase method of $\mathrm{Hu}$ and Adams (2006). It must be noted that in the CLF model, the effect of surface heterogeneity or roughness of the solid is not included, since interfacial tensions are the only input parameters.

The goal of this work is to provide an extension of the CLF model of Huber et al. (2016b) in a way that contact line motion can be simulated satisfactorily also for low capillary numbers when stick-slip effect is dominant. The extended SPH model uses an effective upscaled description of the short-range fluid-solid interactions, represented as a locally increased viscous dissipation, following the formulation of de Gennes et al. (2003). The employed macroscopic model of increased local dissipation at the contact line can be deduced from locally heterogeneous surface energies at the microscale. In Fig. 3, a sketch of an infinitesimally slow wetting process across a smooth surface with a single obstacle is shown. The contact line pins to the defect when the fluid interface moves across the surface. Since the interface gets deformed in the vicinity of the defect (Fig. $3\left(t_{2}\right)$ ), potential energy is stored in the system. After the snap-off event, this potential energy is dissipated in the fluid close to the contact line. From an upscaled perspective, it is still possible that the contact line moves with a constant velocity but the energy or driving pressure gradient, needed to move the interface, is significantly increased. This approach is a natural choice for the SPH method, since dynamic contact angles are not given as a boundary condition, in contrast to the VOF method, but result directly from the equilibrium of the contact line force and the viscous 


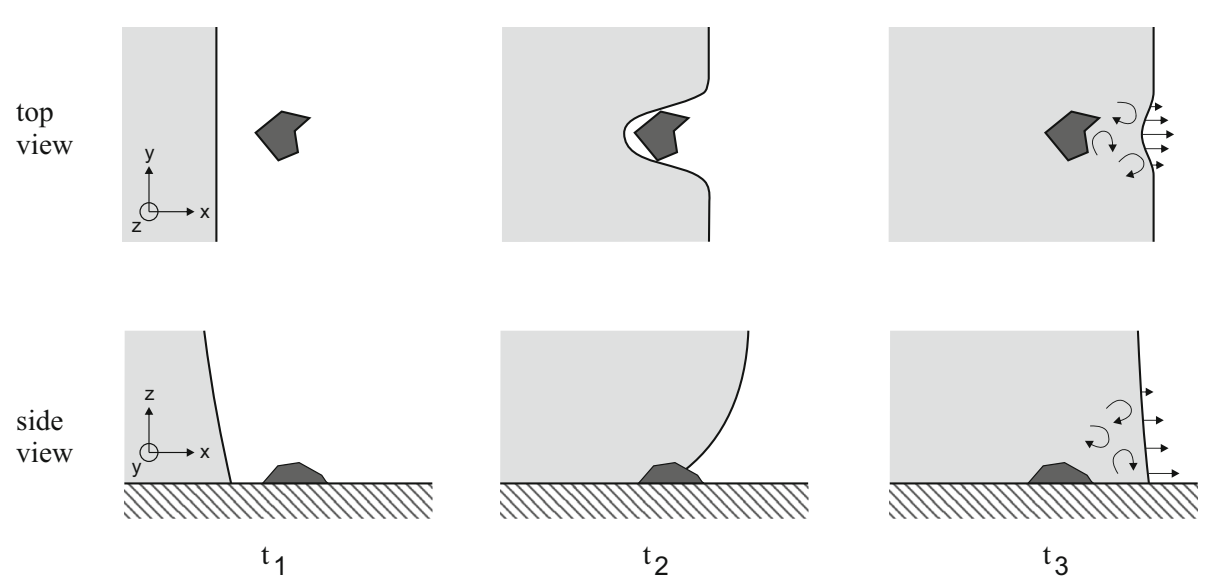

Fig. 3 Microscopic view of local energy dissipation at a single obstacle for an infinitesimal slow moving interface over time. $\left(t_{1}\right)$ Relaxed interface just before pinning. $\left(t_{2}\right)$ Deformation of interface where contact line is pinned to the defect. $\left(t_{3}\right)$ Interface relaxation after snap-off with local kinetic energy dissipation

dissipation close to the contact line. We perform a quantitative comparison of our model with data from wetting experiments on a tape, which are introduced in Sect. 1.2.

\section{Model Description}

Isothermal flow of immiscible and incompressible Newtonian fluids on the pore scale is described by the Navier-Stokes equations. With the extensions for surface tension effects between wetting, non-wetting and solid phase, the Lagrangian formulation of the momentum balance of a fluid element reads (see, e.g., Huber et al. 2016b):

$$
\rho \frac{\mathrm{D} \mathbf{v}}{\mathrm{D} t}=-\nabla p+\mu \Delta \mathbf{v}+\rho \mathbf{g}+\mathbf{F}_{\mathrm{wn}}^{\mathrm{VOL}}+\mathbf{F}_{\mathrm{wns}}^{\mathrm{VOL}},
$$

where the terms $-\nabla p$ and $\mu \Delta \mathbf{v}$ account for pressure and viscous forces in the system, respectively. Further, $\mathbf{g}$ represents any body force like gravity. $\mathbf{F}_{\mathrm{wn}}^{\mathrm{VOL}}$ and $\mathbf{F}_{\mathrm{wns}}^{\mathrm{VOL}}$ are volumetric forces which represent the locally distributed forces acting at the fluid-fluid interface and the forces at the solid-fluids contact line. $\mathbf{F}_{\mathrm{wn}}^{\mathrm{VOL}}$ is given by Brackbill et al. (1992) as:

$$
\mathbf{F}_{\mathrm{wn}}^{\mathrm{VOL}}=\mathbf{f}_{\mathrm{wn}} \delta_{\mathrm{wn}}=\sigma_{\mathrm{wn}} \kappa_{\mathrm{wn}} \mathbf{n}_{\mathrm{wn}},
$$

and is called continuous surface force. Here, $f_{\mathrm{wn}}, \delta_{\mathrm{wn}}, \sigma_{\mathrm{wn}}, \kappa_{\mathrm{wn}}$ and $\mathbf{n}_{\mathrm{wn}}$ are the surface force, Dirac delta distribution, surface tension coefficient, curvature and the normal vector of the interface between the two immiscible phases. The normal vector is calculated using the color (or indicator) function c.

$$
\mathbf{n}_{\mathrm{wn}}=\frac{\nabla c}{[c]},
$$

where $[c]=\left|c_{\mathrm{w}}-c_{\mathrm{n}}\right|$ is the change in $c$ at the interface. $\left|\mathbf{n}_{\mathrm{wn}}\right|$ is used as volume reformulation and approximates the Dirac delta distribution $\delta_{\mathrm{wn}}$, because the condition

$$
\int \mathbf{n}_{\mathrm{wn}} \cdot \mathrm{d} \hat{\mathbf{n}}_{\mathrm{wn}}=1
$$


Fig. 4 Contact line at the connection point of the unit vectors $\hat{v}_{\mathrm{wn}}, \hat{v}_{w s}$ and $\hat{v}_{\mathrm{ns}}$ where each vector is located tangential on the corresponding interface

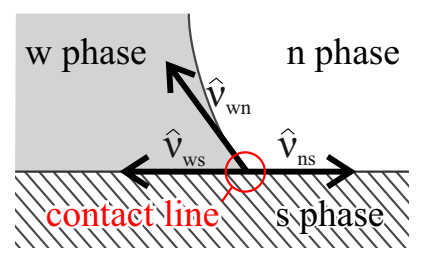

is fulfilled. The local curvature of the interface $\kappa_{\mathrm{wn}}$ is defined as

$$
\kappa_{\mathrm{wn}}=-\nabla \cdot \hat{\mathbf{n}}_{\mathrm{wn}},
$$

where $\hat{\mathbf{n}}_{\mathrm{wn}}$ is the normalized normal vector. The momentum balance at a contact line tangential to the wall, including the volume reformulation, reads:

$$
\mathbf{F}_{\mathrm{wns}}^{\mathrm{VOL}}=\left(\sigma_{\mathrm{ns}}-\sigma_{w s}+\sigma_{\mathrm{wn}}(\underbrace{\hat{v}_{\mathrm{ns}} \cdot \hat{v}_{\mathrm{wn}}}_{-\cos \theta_{\mathrm{D}}})\right) \nu_{\mathrm{ns}} \delta_{\mathrm{wns}},
$$

where $\hat{v}_{k l}$ is a unit vector tangential to the $k l$-interface as shown in Fig. 4 and $\sigma_{k l}$ is the surface tension coefficient between phases $k$ and $l$. In Eq. (6), $\delta_{\mathrm{wns}}$ is the Dirac delta distribution, which distributes the contact line force to a volumetric force preserving the integral quantity of the force. Under equilibrium conditions, the contact line force vanishes, since the dynamic contact angle $\theta_{\mathrm{D}}$ becomes equal to the static contact angle $\theta_{\mathrm{S}}$ and Eq. (6) reduces to Young's equation:

$$
\sigma_{\mathrm{ns}}=\sigma_{w s}+\sigma_{\mathrm{wn}} \cos \left(\theta_{\mathrm{S}}\right) .
$$

For incompressible fluids, the continuity equation is given as:

$$
\frac{\mathrm{D} \rho}{\mathrm{D} t}=-\rho(\nabla \cdot \mathbf{v})=0 .
$$

In this work, it will be shown that the existing CLF model of Huber et al. (2016b), as given in Eqs. (1) and (8), is able to find dynamic contact angles that are in good agreement with experimental observations for wetting, but only at high capillary numbers on homogeneous surfaces. In such cases, the contact line dynamics are mainly determined by the viscous dissipation in the liquid. This was also observed by Blake (2012) when comparing the wetting behavior of water on gelatin-coated and uncoated PET. For low capillary numbers, that model has to be extended, as explained below.

\subsection{Stick-Slip Model: Extended CLF Model Including Fluid-Solid Interactions at the Contact Line}

For low capillary numbers, solid-liquid interactions modify the dynamic contact angle significantly such that it cannot be captured with the CLF model. Therefore, an upscaled model of various molecular solid-liquid interactions (see Fig. 1) is introduced. The main idea is to account for those microscopic effects at the contact line by modifying the viscous term in the momentum balance of a fluid element [see Eq. (1)] in the vicinity of the contact line. The modified force is able to dominate the dynamics at low capillary numbers, as found in experiments by Blake (1993).

Using the idea of local energy dissipation by viscous forces in the liquid after snap-off at a sparse defect (de Gennes et al. 2003), the existing model is extended by introducing a variable viscosity $\mu_{\text {SSL }}$ in the vicinity of the contact line to account for stick-slip (SSL) 

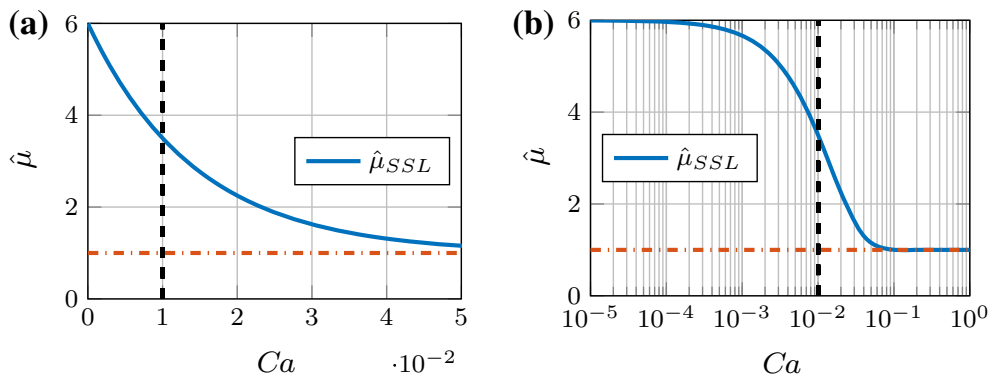

Fig. 5 Plot of the dimensionless viscosities of the stick-slip model (SSL) for $\alpha=5$ and $\mathrm{Ca}_{\mathrm{Tp}}=10^{-2}$ given in normal (a) and logarithmic (b) scale. For comparison, the unaltered viscosity of the CLF model of Huber et al. (2016b) is plotted in the red dash-dotted line

behavior. This viscosity replaces the viscosity parameter in Eq. (1), for interactions with the solid phase. It is assumed to be a decreasing function of capillary number:

$$
\mu_{\mathrm{SSL}}=\mu\left(1+\alpha(1 / 2)^{\left(C a / C a_{\mathrm{Tp}}\right)}\right) \text {, with } C a=\frac{\mu\left|\mathbf{v}_{\mathrm{cl}}\right|}{\sigma_{\mathrm{wn}}},
$$

where $\mathbf{v}_{\mathrm{cl}}$ is the contact line velocity. Hereby, the viscosity function $\mu_{\mathrm{SSL}}$ is used in the same volume where the force at the contact line is distributed. This model introduces two parameters $\alpha$ and $C a_{\mathrm{Tp}}$ for the fluid-solid interactions. In Eq. (9), $\alpha$ is a parameter for the maximum fractional increase in viscosity at the contact line with respect to the bulk viscosity. $C a_{\mathrm{Tp}}$ regulates the transition between the slow and fast wetting regimes. For larger values of $C a_{\mathrm{Tp}}$, stick-slip behavior is apparent in faster wetting regimes.

In Fig. 5, the dimensionless viscosity $\hat{\mu}_{\mathrm{SSL}}=\mu_{\mathrm{SSL}} / \mu$ is plotted as a function of the capillary number with chosen values $\alpha=5$ and $C a_{\mathrm{Tp}}=10^{-2}$. The plot is given in normal and logarithmic scales to highlight the behavior at low capillary numbers. Additionally, the capillary number at the transition point $C a_{\mathrm{Tp}}$ between the two different wetting modes is marked by a dashed line. $C a_{\mathrm{Tp}}$ is defined as the capillary number of the wetting process, where the viscosity increase at the contact line is exactly half of the maximum increase. We provide explanation on how to choose the values of $C a_{\mathrm{Tp}}$ and $\alpha$ in Sect. 4.1. A qualitative discussion on the effect of the $\mathrm{Ca}$-dependent viscosity increase on the energy dissipation is given in "Appendix A".

\subsection{Implementation in SPH}

We chose the Lagrangian SPH method because of its mesh-free nature and the straightforward treatment of interface movement. SPH is an interpolation method where properties are evaluated at certain interpolation points to approximate a continuous field. These points, which represent a certain volume, are called particles. In SPH, the interpolation formula for any quantity $A$ of a particle $i$ at the position $\mathbf{r}_{i}$ is an approximation to an integral interpolant of the form:

$$
A\left(\mathbf{r}_{i}\right)=\int A\left(\mathbf{r}^{\prime}\right) W\left(\mathbf{r}-\mathbf{r}^{\prime}, h\right) \mathrm{d} \mathbf{r}^{\prime} \approx \sum_{j}^{N_{i}} \frac{m_{j} A_{j}}{\rho_{j}} W_{i j},
$$

where $m_{j}$ and $\rho_{j}$ are the mass and the density of a neighboring particle $j$, respectively. $W_{i j}$ is the short form of $W\left(\mathbf{r}_{i}-\mathbf{r}_{j}, h\right)$ which is the kernel function evaluated for the distance 
between particles $i$ and $j$ and a smoothing length $h$, which we set to 2.1 times the initial particle spacing $\Delta x$ for the presented simulations in this work. For the choice of $h$ in 2D simulations of two-phase flow, we refer to Szewc et al. (2012). The upper bound of summation $N_{i}$ is the number of neighboring particles of the center particle $i$ (including particle $i$ itself) for which a positive value of the kernel function exists; $W_{i j}>0$. To simplify the notation, $N_{i}$ is omitted in the following equations. We use the incompressible SPH method, introduced by Cummins and Rudman (1999). As kernel function, we take the C2 spline function of Wendland (1995) for 2D systems:

$$
W(\mathbf{r}, h)=\frac{7}{4 \pi h^{2}} \begin{cases}\left(1-\frac{q}{2}\right)^{4}(2 q+1) & \text { if } q<2 \\ 0 & \text { else },\end{cases}
$$

where $q=|\mathbf{r}| / h$. A detailed introduction to the SPH theory can be found, for example, in the review of Monaghan (2011).

\subsubsection{SPH Formulation for Multi-phase Flow}

To overcome deficiencies of the particle distribution at open boundaries , and for a more accurate calculation of unit vectors normal to the interface close to the contact line, we use the "corrected gradient of the corrected kernel" $\widetilde{\nabla}_{i} \widetilde{W}_{i j}$ as introduced by Bonet and Lok (1999) for all computed field variables. The corrected kernel is defined as:

$$
\tilde{W}_{i j}=\frac{W_{i j}}{\sum_{j} V_{j} W_{i j}}=\frac{W_{i j}}{S_{i}},
$$

with $\tilde{W}_{i j}=\tilde{W}\left(\mathbf{r}_{i j}, h\right)=\tilde{W}\left(\mathbf{r}_{i}-\mathbf{r}_{j}, h\right), r_{i j}=\left|\mathbf{r}_{i}-\mathbf{r}_{j}\right| . V_{j}=m_{j} / \rho_{j}$ is the volume of particle $j$ with $m_{j}$ and $\rho_{j}$ being its mass and density, respectively. $S_{i}$ is called the Shepard function. The gradient of this corrected kernel becomes:

$$
\nabla \tilde{W}_{i j}=\nabla\left(\frac{W_{i j}}{S_{i}}\right)=\frac{\nabla W_{i j}-\frac{W_{i j} \nabla S_{i}}{S_{i}}}{S_{i}},
$$

with $\nabla S_{i}=\sum_{j} V_{j} \nabla W_{i j}$. To ensure that the gradient of any linear velocity field is exactly evaluated, the following condition must be fulfilled:

$$
\sum_{j} V_{j} \nabla \tilde{W}_{i j} \otimes \mathbf{x}_{j}=\mathbf{I},
$$

with $\mathbf{x}_{j}$ and $\mathbf{I}$ being the position of particle $j$ and the identity matrix, respectively. Thus, the corrected gradient of the corrected kernel reads:

$$
\tilde{\nabla} \tilde{W}_{i j}=\mathbf{L}_{i} \nabla \tilde{W}_{i j},
$$

with

$$
\mathbf{L}_{i}=\left(\sum_{j} V_{j} \nabla \tilde{W}_{i j} \otimes \mathbf{x}_{j}\right)^{-1}
$$


We use the SPH discretization for multi-phase flow with particle-averaged spatial derivatives (Hu and Adams 2006). Density is computed by

$$
\rho_{i}=m_{i} \sum_{j} W_{i j}
$$

In this work, wall ghost particles (Morris et al. 1997) are used to achieve a no-slip boundary condition at the solid wall. The force per unit mass due to viscosity is then formulated as:

$$
\begin{aligned}
\left(\frac{\mu}{\rho} \Delta \mathbf{v}\right)_{i}= & \frac{1}{m_{i}} \sum_{j}^{N_{i, \text { fluid }}} \bar{\mu}_{i j}\left(V_{i}^{2}+V_{j}^{2}\right) \frac{\mathbf{v}_{i j}}{r_{i j}} \frac{\partial \tilde{W}_{i j}}{\partial r_{i j}} \\
& +\frac{1}{m_{i}} \sum_{j}^{N_{i, \text { solid }}} \mu_{\mathrm{SSL}, i}\left(V_{i}^{2}+V_{j}^{2}\right) \frac{\beta_{i j} \mathbf{v}_{i}}{r_{i j}} \frac{\partial \tilde{W}_{i j}}{\partial r_{i j}}
\end{aligned}
$$

with $\mathbf{v}_{i j}=\mathbf{v}_{i}-\mathbf{v}_{j}$ and

$$
\bar{\mu}_{i j}=\frac{2 \mu_{i} \mu_{j}}{\mu_{i}+\mu_{j}} .
$$

Here, $\mu_{i}$ is the dynamic viscosity of particle $i$ and $\mu_{\mathrm{SSL}}$ was defined in Eq. 9. The indices fluid and solid denote an exclusive summation over neighboring fluid or solid particles. If the stick-slip model is not applied, the viscosity of particle $i$ simplifies to $\mu_{\mathrm{SSL}, i}=\mu_{i}$ which is then identical to the original form defined by Morris et al. (1997). According to Morris et al. (1997), the factor $\beta_{i j}$ in Eq. 18 is given as:

$$
\beta_{i j}=1+\frac{d_{j}}{d_{i}}
$$

where $d_{i}$ and $d_{j}$ are the distance of particle $i$ and $j$ in the direction normal to the solid-fluid interface. The force per unit mass due to the pressure field gradient reads:

$$
-\left(\frac{1}{\rho} \nabla p\right)_{i}=-\frac{1}{m_{i}} \sum_{j} \bar{p}_{i j}\left(V_{i}^{2}+V_{j}^{2}\right) \tilde{\nabla}_{i} \tilde{W}_{i j},
$$

with

$$
\bar{p}_{i j}=\frac{\rho_{i} p_{j}+\rho_{j} p_{i}}{\rho_{i}+\rho_{j}}
$$

We use the formulation of Adami et al. (2010) for the computation of the normal vectors at the fluid-fluid interface for the computation of the continuous surface force (Eq. 2):

$$
\mathbf{n}_{\mathrm{wn}, i}=\frac{1}{|\nabla c| V_{i}} \sum_{j} \bar{c}_{i j}\left(V_{i}^{2}+V_{j}^{2}\right) \tilde{\nabla}_{i} \tilde{W}_{i j}
$$

with

$$
\bar{c}_{i j}=\frac{\rho_{j}}{\rho_{i}+\rho_{j}} c_{i}^{i}+\frac{\rho_{i}}{\rho_{i}+\rho_{j}} c_{j}^{i}
$$

where

$$
c_{l}^{k}= \begin{cases}1, & \text { if the phases of particles } k \text { and } l \text { are different } \\ 0, & \text { if the phases of particles } k \text { and } l \text { are the same }\end{cases}
$$


The curvature in our SPH model is computed as:

$$
\kappa_{\mathrm{wn}, i}=-\sum_{j} \frac{m_{j}}{\rho_{j}}\left(\hat{\mathbf{n}}_{\mathrm{wn}, j}-\hat{\mathbf{n}}_{\mathrm{wn}, i}\right) \cdot \tilde{\nabla}_{i} \tilde{W}_{i j}
$$

From this, the surface force is given as a volumetric force:

$$
\mathbf{F}_{\mathrm{wn}, i}^{\mathrm{VOL}}=\sigma_{\mathrm{wn}} \kappa_{\mathrm{wn}, i} \mathbf{n}_{\mathrm{wn}, i}
$$

The volume reformulation of the contact line force (Eq. 6) is computed by

$$
\delta_{\mathrm{wns}, i}=-\zeta \hat{\mathbf{d}}_{j} \cdot \sum_{j}\left(\delta_{\mathrm{wns}, j}^{\prime}-\delta_{\mathrm{wns}, i}^{\prime}\right) \widetilde{\nabla}_{i} \tilde{W}_{i j}
$$

where

$$
\delta_{\mathrm{wns}, j}^{\prime}= \begin{cases}\delta_{\mathrm{wns}, i}^{\prime}, & \text { if } j \in N_{i, \text { fluid }} \\ 0, & \text { if } j \in N_{i, \text { solid }}\end{cases}
$$

and

$$
\delta_{\mathrm{wns}, i}^{\prime}=v_{\mathrm{ns}, i} \cdot \mathbf{n}_{\mathrm{wn}, i}
$$

with

$$
v_{\mathrm{ns}, i}=\left|\mathbf{d}_{i}\right|^{2} \mathbf{n}_{i}-\left(\mathbf{d}_{i} \cdot \mathbf{n}_{i}\right) \mathbf{d}_{i}
$$

In Eq. 27, $\hat{\mathbf{d}}_{j}$ is the normalized distance vector pointing toward the closest solid phase boundary. In the work of Huber et al. (2016b), the factor $\zeta$ is set as 2, because the volume reformulation is only evaluated in the fluid domain and it is assumed that all forces present at the contact line are exerted to the fluid phase. Contrary to the formulation of the CLF model (Huber et al. 2016b), a summation, weighted by the volume reformulation term $\delta_{\text {wns }}$, is used for a more homogeneous calculation of the contact angle at the contact line.

$$
-\cos \theta_{D, i}=\sum_{j} \frac{m_{j} / \rho_{j} \delta_{\mathrm{wns}, j} \hat{\mathbf{d}}_{j} \cdot \hat{\mathbf{n}}_{\mathrm{wn}, j}}{m_{j} / \rho_{j} \delta_{\mathrm{wns}, j}},
$$

where $\theta_{D, i}, \hat{\mathbf{d}}_{j}$ and $\hat{\mathbf{n}}_{\mathrm{wn}, j}$ are the dynamic contact angle, the normalized distance vector pointing toward the closest solid phase boundary and the interface unit normal vector pointing toward the non-wetting phase, respectively. Thus, the local contact line force is computed by:

$$
\mathbf{F}_{\mathrm{wns}, i}^{\mathrm{VOL}}=\sigma_{\mathrm{wn}}\left(\cos \theta_{\mathrm{S}}-\cos \theta_{D, i}\right) \hat{v}_{\mathrm{ns}, i} \delta_{\mathrm{wns}, i}
$$

A second modification to the CLF model used by Huber et al. (2016b) was a boundary condition for $\mathbf{F}_{\mathrm{wn}, i}^{\mathrm{VOL}}$ (see Eq. (2)). It was experienced that the computation of the interface curvature $\kappa_{i}$ is quite error-prone close to a solid phase. Due to numerical artifacts, as a consequence of missing support of neighboring particles at the boundary, the curvature $\kappa_{i}$ is likely to change its sign at the boundary. Therefore, the boundary condition for $\mathbf{F}_{\mathrm{wn}}^{\mathrm{VOL}}$ was chosen to be:

$$
\left.\mathbf{F}_{\mathrm{wn}}^{\mathrm{VOL}}\right|_{\left|\mathbf{d}_{i}\right|=0}=0
$$


$\sim \mathrm{VOL}$

This condition is enforced by multiplying the original continuous surface force $\widetilde{F}_{\mathrm{wn}, i}$ with a transition function $S_{i}$

$$
\mathbf{F}_{\mathrm{wn}, i}^{\mathrm{VOL}}=\widetilde{\mathbf{F}}_{\mathrm{wn}, i}^{\mathrm{VOL}} B_{i}
$$

with

$$
\begin{aligned}
B_{i} & =\frac{b_{1, i}-b_{2, i}}{s_{1, i}+b_{2, i}} \\
b_{1, i} & =\sum_{j}^{N_{i, \text { fluid }}} m_{j} / \rho_{j} \frac{\partial W}{\partial r_{i j}} \\
b_{2, i} & =\sum_{j}^{N_{i, \text { solid }}} m_{j} / \rho_{j} \frac{\partial W}{\partial r_{i j}},
\end{aligned}
$$

where $\frac{\partial W}{\partial r_{i j}}$ is the kernel derivative. The indices fluid and solid denote an exclusive summation over neighboring fluid or solid particles, respectively.

Since the SPH scheme used in this work differs from the scheme of Hu and Adams (2006), we present simulations of a Taylor-Green vortex in "Appendix B" to show the good accuracy and convergence of our scheme. A detailed introduction into the treatment of open boundaries is given in the work of Kunz et al. (2016a).

\subsection{Stick-Slip Model in SPH}

A modification to the model is needed for the local assignment of viscosity, which is not constant, when the stick-slip model is employed (see Eq. 9). Therefore, the local capillary number of particle $i$ at the contact line including the contact line velocity is determined by:

$$
C a_{i}=\frac{\mu_{i}\left|\mathbf{v}_{c l, i}\right|}{\sigma_{\mathrm{wn}}}
$$

with:

$$
\mathbf{v}_{c l, i}=\frac{\sum_{j} V_{j} \delta_{\mathrm{wns}, j} \mathbf{v}_{j}}{\sum_{j} V_{j} \delta_{\mathrm{wns}, j}}
$$

where $\delta_{\mathrm{wns}, j}$ is the volume reformulation term of a neighboring particle $j$ for the contact line force. A detailed description of the computation of the volume reformulation term $\delta_{\mathrm{wns}}$ is given by Huber et al. (2016b). The computed capillary number for particle $i$ (Eq. 38) then is used for the calculation of the stick-slip viscosity of particle $i$ :

$$
\mu_{\mathrm{SSL}, i}= \begin{cases}\mu_{i}\left(1+\alpha(1 / 2)^{\left(C a_{i} / C a_{\mathrm{Tp}}\right)}\right), & \text { if } \delta_{\mathrm{wns}, i}>0 \\ \mu_{i}, & \text { if } \delta_{\mathrm{wns}, i}=0\end{cases}
$$

According to Eq. (40), a viscosity increase is applied only in the vicinity of the contact line, where the force at the contact line from the CLF model is present. 


\section{Application of the CLF Model for Two-Phase Flow Simulations}

\subsection{Evaluation of the Volume Reformulation Term of the CLF Model}

Huber et al. (2016b) showed for the CLF model that the error in the computed equilibrium contact angles for sessile drops was below $5 \%$ in the partially wetting regimes between $30^{\circ}<\theta_{\mathrm{E}}<140^{\circ}$. A sketch of the initial phase configuration for all simulations is given in Fig. 6. Besides the capability, needed to capture correct equilibrium contact angles, a fundamental requirement of the CLF model is the volume reformulation term $\delta_{\text {wns }}$ at the contact line which must satisfy the following condition:

$$
\int \delta_{\text {wns }} \mathrm{d} \mathbf{r}=1
$$

The validity of this condition has to be shown. The validation was done with the same set of simulations of sessile drops as employed by Huber et al. (2016b) for the verification of equilibrium contact angles. The resolution for the two-phase simulations was set to $200 \times 100$ particles. The error in meeting restriction (41) for a wide range of equilibrium contact angles is shown in Fig. 7. With an error of less than $\pm 1 \%$ for $30^{\circ}<\theta_{\mathrm{E}}<150^{\circ}$, the CLF model is well suited to simulate dynamic contact angles in this work.

\subsection{Comparison of the Dynamic Contact Angles Computed by CLF Model with Experimental Data}

In this section, the CLF model of Huber et al. (2016b) is employed to calculate dynamic contact angles for a wide range of capillary numbers in a system similar to experiments of Blake and Shikhmurzaev (2002) shown in Fig. 2. We only consider the piece of tape in Fig. 2 that enters the liquid. The system we are modeling is shown in Fig. 8. The tape is modeled as rigid plate moving downward. The non-wetting fluid represents the air, and the wetting fluid is the solution in Fig. 2. The length of the system is chosen to be $l=3 d$, where $d$ is the domain width, in order to satisfy a parallel flow profile at the open boundaries. The resolution for all

Fig. 6 Sketch of the initial configuration of the liquid droplets on a solid wall

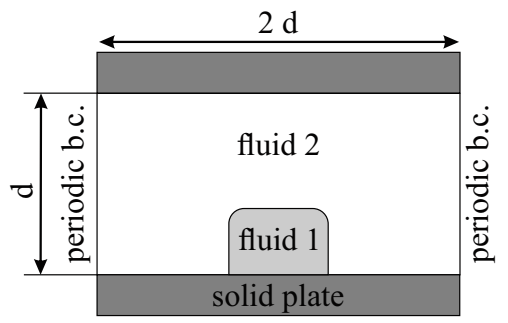

Fig. 7 Absolute error and standard deviation of the integral volume reformulation with respect to the set contact angle

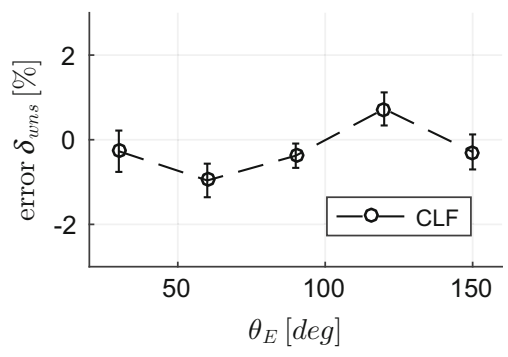


Fig. 8 Simulation setup to capture dynamic contact angles in a wide range of wetting dynamics

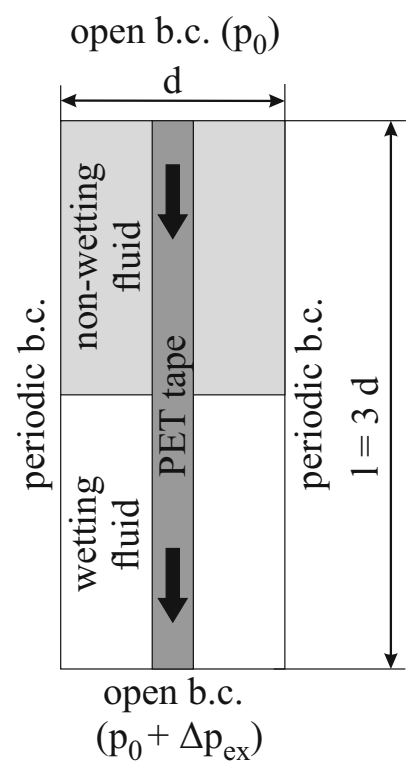

Table 1 Fluid properties used for dynamic wetting simulations

\begin{tabular}{lclllr}
\hline$\%(\mathrm{glyc})$ & $\mu(\mathrm{mPas})$ & $\sigma_{l a}(\mathrm{~N} / \mathrm{m})$ & $\theta_{0}\left({ }^{\circ}\right)$ & $\rho\left(\mathrm{kg} / \mathrm{m}^{3}\right)$ & $d(\mathrm{~mm})$ \\
\hline 0 & 1 & 0.0728 & 82.0 & 1000 & 1 \\
59 & 10 & 0.0653 & 64.5 & 1150 & 10 \\
86 & 104 & 0.0658 & 65.0 & 1224 & 10 \\
\hline
\end{tabular}

simulations was set to $300 \times 100$ particles. A resolution study for one case is shown at the end of this section. As in experiments of Blake and Shikhmurzaev (2002) and Blake (2012), three different solutions were used in different experiments. Properties of the solutions used in various simulations are given in Table 1 . These are either pure water (as used in experiments of Blake and Shikhmurzaev (2002)) or a mix of water and glycerol (as used in experiments of Blake (2012)). In that table, also the domain width $d$ is given. For computational convenience, the non-wetting phase was assigned to have exactly the same properties as the wetting phase in each simulation. In general, SPH can be used to simulate liquid-air systems with the SPH scheme of Adami et al. (2010). The only reason we have chosen the same fluid properties was the significantly increased spurious currents for a liquid-air system, which had an adverse influence on the computation of the contact line velocity (Eq. 39). Of course, the phases were assumed to be immiscible with an interfacial tension given in Table 1. To avoid that a fluid is driven out of the simulation domain by the moving plates, a variable pressure difference $\Delta p_{\text {ex }}$ was imposed between the open boundaries at top and bottom. This allowed us to keep the fluid-fluid interface in the middle of the domain and the initial phase fractions constant. Thus, the applied pressure difference $\Delta p_{\text {ex }}$ was changed in every time step according to the following pressure proportional-integral (PI) control formula: 
Table 2 PI controller parameters used in the simulations

\begin{tabular}{lcclcc}
\hline$\%$ (glyc) & \multicolumn{1}{c}{$K_{\mathrm{p}}$} & $K_{\mathrm{i}}$ & $K_{\mathrm{f}}$ & $\Delta p_{\text {ex }, \min }$ & $\Delta p_{\text {ex }, \max }$ \\
\hline 0 & 800 & 20 & 13.7 & -400 & 100 \\
59 & 800 & 10 & 5.6 & -430 & 10 \\
86 & 1000 & 30 & 5.6 & -4200 & 100 \\
\hline
\end{tabular}

$$
\Delta p_{\mathrm{ex}}=\underbrace{K_{\mathrm{p}} \overline{\mathbf{v}}_{\text {in }}}_{=P}+\underbrace{K_{\mathrm{i}} \int_{0}^{t} \overline{\mathbf{v}}_{\text {in }} \mathrm{d} t}_{=I}+\underbrace{K_{\mathrm{f}}}_{=F} .
$$

The calculated value of $\Delta p_{\text {ex }}$ was limited to stay within lower and upper bounds $\Delta p_{\text {ex }}$, min and $\Delta p_{\mathrm{ex}, \max }$. According to the bounds of $\Delta p_{\mathrm{ex}}$, the integral term $I$ is adjusted if necessary:

$$
I= \begin{cases}\Delta p_{\mathrm{ex}, \max }-P-F & , \text { if } \Delta p_{\mathrm{ex}}>\Delta p_{\mathrm{ex}, \max } \\ \Delta p_{\mathrm{ex}, \text { min }}-P-F & , \text { if } \Delta p_{\mathrm{ex}}<\Delta p_{\mathrm{ex}, \text { min }}\end{cases}
$$

The corresponding controller parameter values are given in Table 2.

The applicability and robustness of such a pressure control have been shown by Kunz et al. (2016a).

The dynamic wetting behavior of the CLF model was studied and compared with the experimental data of Blake and Shikhmurzaev (2002) and Blake (2012) over a wide range of viscosity values. In Fig. 9, the comparison between CLF simulations and the experimental data is presented. The results are given in linear scale (left column) and logarithmic scale (right column). In logarithmic scale, the discrepancy between simulation results and experimental data for slow dynamics is highlighted, where in the linear scale the linear behavior for fast dynamics is more apparent. The results for 59 and $86 \%$ aqueous glycerol solutions are also compared to a semiempirical model of Shikhmurzaev (1997) which takes information of the contact line speed and the flow field in the vicinity of the contact line into account. The model parameters are the same as used by Blake and Shikhmurzaev (2002) which have been fitted once for the whole series of aqueous glycerol solutions.

The CLF simulations of all three fluid systems have some properties in common:

1. All CLF simulations reproduce the equilibrium contact angle very well $\mathrm{Ca}=0)$.

2. The computed dynamic contact angles show an almost linear dependency on the contact line velocity (or $\mathrm{Ca}$ ) in the investigated range (see linear plots of Fig. 9).

3. The results for 59 and $86 \%$ aqueous glycerol solutions match the results of the semiempirical model of Shikhmurzaev (1997) quite good. Since the flow field at the contact line is resolved in the SPH simulations, the dependency of the dynamic contact angle on the fluid viscosity is captured in the CLF model as predicted by the semiempirical model of Shikhmurzaev (1997).

4. Only for the case of pure water (Figs. 9a, b), there is a reasonable agreement between simulated and measured contact angles for $C a>1 \times 10^{-3}$. But, for slower dynamics, simulated contact angles are significantly smaller.

5. For 59 and $86 \%$ aqueous glycerol solutions, simulated values are significantly smaller than measured values for the whole range of capillary numbers.

With the choice of a liquid-liquid system in the SPH simulations, it is likely that the damping effect is overestimated by a factor of two compared with a gas-liquid combination, which is the case in the experiments. For the case of considerably large wetting speeds of $\mathrm{Ca}>10^{-2}$, 

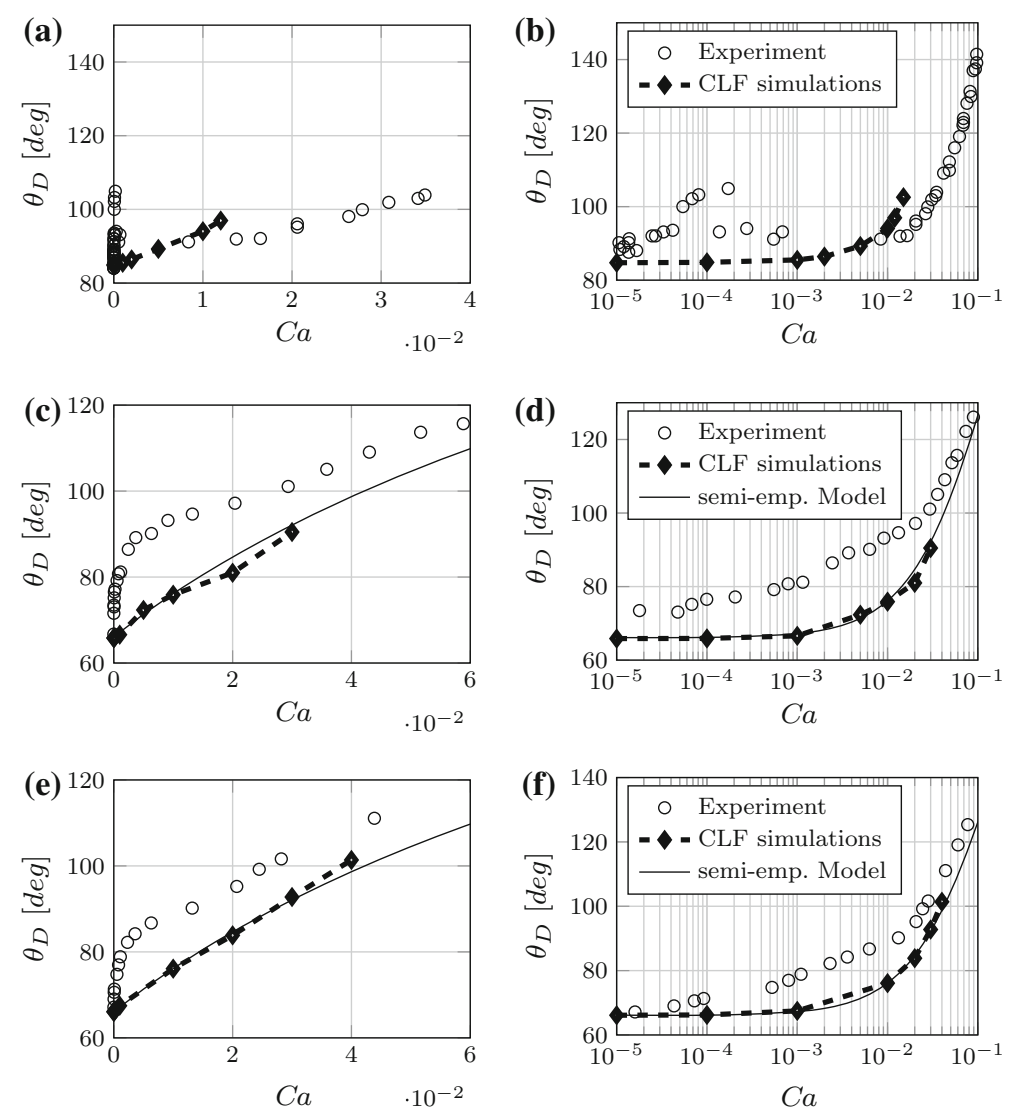

Fig. 9 Comparison of SPH simulations with the CLF model and experiments of dynamic wetting processes with different wetting liquids on a PET tape. a, b Water on PET, c, d 59\% aqueous glycerol solution, e, f $86 \%$ aqueous glycerol solution. The results for 59 and $86 \%$ aqueous glycerol solution are also compared to the model of Shikhmurzaev (1997)

the influence of the choice of $\zeta=2$ in the volume reformulation term (Eq. 27) should be studied more closely. Indeed, it seems questionable that all the forces at the contact line work only on the fluid phases, as stated by Huber et al. (2016b). The large differences between simulated and measured values show that some major effect is not included in CLF model. This is more evident in the case of low capillary numbers, where fluid-solid interactions play a crucial role as stated by Blake (1993).

\section{Application of the Stick-Slip (SSL) Model for Two-Phase Flow Simulations}

\subsection{Comparison of the Dynamic Contact Angles Computed by the SSL Model with Experimental Data}

In order to show that the stick-slip (SSL) model properly simulates the full range of wetting dynamics, it was employed to simulate the same three cases described in the previous section. 
Table 3 Stick-slip parameters for liquid-solid interactions

\begin{tabular}{lll}
\hline$\%$ (glyc) & $\alpha$ & $C a_{\mathrm{Tp}}$ \\
\hline 0 & 227 & $1.91 \times 10^{-4}$ \\
59 & 9.07 & $5.78 \times 10^{-3}$ \\
86 & 4.5 & $7.30 \times 10^{-3}$ \\
\hline
\end{tabular}
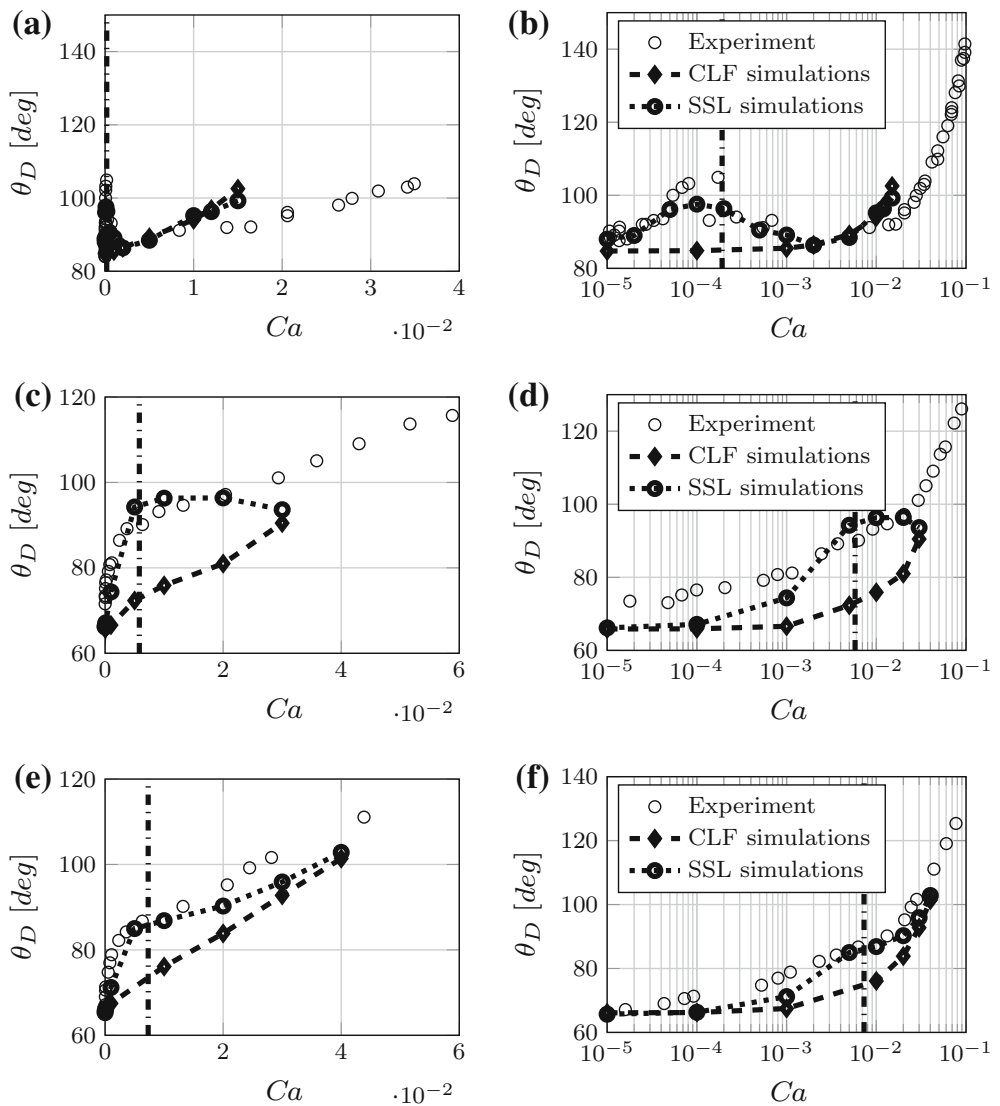

Fig. 10 Comparison of SPH simulations with the SSL model and experiments of dynamic wetting processes with different wetting liquids on a PET tape. Vertical dash-dot lines are representing the capillary number at the transition point $C a_{\mathrm{Tp}}$ a, b water on PET, c, d 59\% aqueous glycerol solution, e, $\mathbf{f} 86 \%$ aqueous glycerol solution

The proper choice of the two parameters is clearly crucial for the simulated physical effect of stick-slip behavior. A good choice of $C a_{\mathrm{Tp}}$ comes directly from experimental results. $C a_{\mathrm{Tp}}$ is in the region where the offset in dynamic contact angles between experiments and SPH simulations without the stick-slip extension becomes smaller. A good choice of $\alpha$ is the ratio between the initial slope of dynamic contact angle with respect to wetting speed comparing SPH simulations without the stick-slip extension and experimental data. The parameters $\alpha$ and $C a_{\mathrm{Tp}}$ for the three liquids wetting PET are given in Table 3.

In Fig. 10, the results of the SPH simulations using the new SSL model are compared with the experimental data as well as SPH simulations using the CLF model. It is shown 
(a)

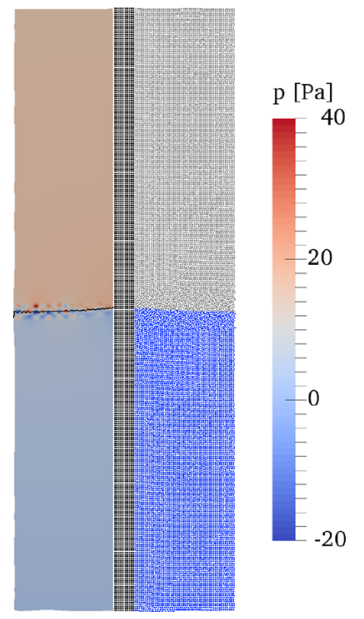

(b)

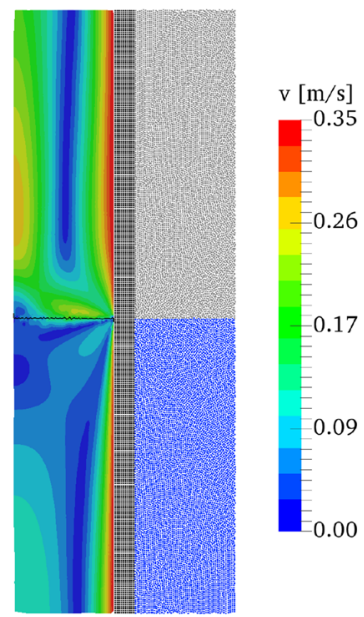

(c)

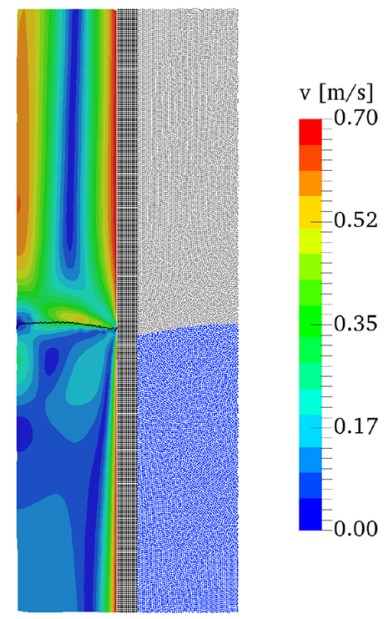

Fig. 11 Particle snapshots for $C a=0(\mathbf{a}), C a=5 \times 10^{-3}(\mathbf{b})$ and $C a=1 \times 10^{-2}$ (c) at $t^{*}=6$. For $C a=0$ also the pressure field is shown on the left half of the plot and for $C a=5 \times 10^{-3}$ and $C a=1 \times 10^{-2}$ the velocity field is given

that the dynamic contact angles of the SSL simulations match the experimental values over a wide range of capillary numbers. The effect of the two different wetting modes with different dominant dissipation sources (viscous forces or fluid-solid interactions), as discussed in Sect. 1.2, on the dynamic contact angle is different for pure water and aqueous glycerol solutions. In the case of pure water, the dynamics of the biggest influence of the two wetting modes on the dynamic contact angle are clearly separated (see Fig. 10b). Following the qualitative discussion on the behavior of the SSL model in "Appendix A", effects of fluidsolid interactions on $\theta_{\mathrm{D}}$ are dominant up to $C a \approx 1 \times 10^{-3}$, while effects from viscous dissipation in the flow field close to the contact line are significant effect after $C a \approx 5 \times 10^{-3}$. In the case of aqueous glycerol solutions, viscous forces and fluid-solid interactions affect the dynamic contact angle for $C a \approx 1 \times 10^{-2}$ with similar intensity (see Fig. 10d, f). It is clear that the stick-slip model results agree quite well with measurements in both cases. Particle snapshots obtained with our stick-slip model are shown in Fig. 11. In Fig. 11 (a), the pressure field inside the phases on the left side of the tape is shown, as while the particle snapshot at $t=1 \mathrm{~s}$ is shown on the right side. Inside the phases, the pressure field is homogeneous as is expected when using ISPH. Nevertheless, some pressure oscillations are apparent close to the fluid-fluid interface, which correspond to spurious currents as a result of the applied continuous surface force. In Fig. $11 \mathrm{~b}$, c, the velocity fields at the dimensionless simulation time $t^{*}=\frac{t v_{W}}{l}=6$ are shown for the cases $C a=5 \times 10^{-3}$ and $C a=1 \times 10^{-2}$, respectively. In Fig. 12, the convergence of the computed steady-state dynamic contact angles is shown. This point is crucial, since it shows that the balance between the force at the contact line and viscous dissipation is not affected with increasing resolution, even though the force at the contact line is applied in a smaller area closer to the solid wall. Also, the parameters of the stick-slip model are invariant to the chosen resolution. For a good reproduction of the equilibrium contact angles, we chose $N_{d}=100$ in all our simulations. 

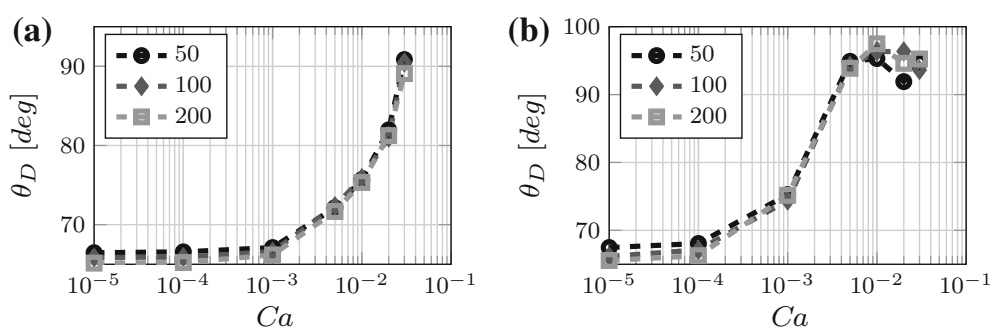

Fig. 12 The simulation results for different domain resolutions is shown for the case of 59\% aqueous glycerol solutions for the case without the stick-slip model (a) and with the stick-slip model (b). The legend entries correspond to the initial number of particles over the domain width $d$

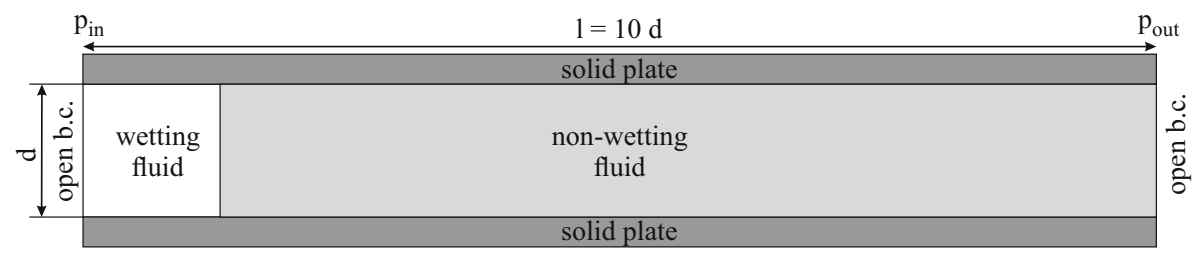

Fig. 13 Sketch of the two plates being wetted with constant pressure differences $\Delta p_{\text {ex }}$ applied

Table 4 Pressure differences for wetting process with full

\begin{tabular}{lc}
\hline$C a$ & $\Delta p_{\text {ex }}(\mathrm{Pa})$ \\
\hline $1 \times 10^{-3}$ & -50 \\
$2 \times 10^{-3}$ & 38 \\
$5 \times 10^{-3}$ & 300 \\
$1 \times 10^{-2}$ & 736 \\
\hline
\end{tabular}

\subsection{Simulation of Reduced Wetting Dynamics with the Stick-Slip Model at Low Capillary Numbers}

In this section, the influence of fluid-solid interactions around the contact line on the wetting dynamics at low $\mathrm{Ca}$ is shown. We do this by means of simulating the imbibition process between two plates, as shown in Fig. 13, using the SSL model. For the simulations, hydrophilic PET is chosen as the solid phase. The two fluids have the properties of pure water (see Table 1), but they are assumed to be immiscible. The simulation domain consists of two fixed parallel plates separated by a distance of $d=100 \mu \mathrm{m}$. The domain length is $l=10 \mathrm{~d}$. A pressure difference $\Delta p_{\text {ex }}=p_{\text {in }}-p_{\text {out }}$ is imposed across the modeling domain, with four different values (see Table 4) to simulate four different dynamic cases. We assume that the two fluids are immiscible but have the same properties. Then, the averaging gives us the following global momentum balance equation:

$$
\rho l \frac{\mathrm{d} v_{\mathrm{cl}}}{\mathrm{d} t}=\Delta p_{\mathrm{ex}}-\Delta p_{\mathrm{visc}}+p_{\mathrm{c}}
$$

with $\Delta p_{\text {visc }}$ and $p_{\mathrm{c}}$ being the pressure drops caused by viscous dissipation and the capillary pressure, respectively. We assume that there is a Poiseuille flow profile, contact line velocity is equal to the average flow velocity, and there is a static contact angle $\theta_{0}$. As a result, we 
(a)

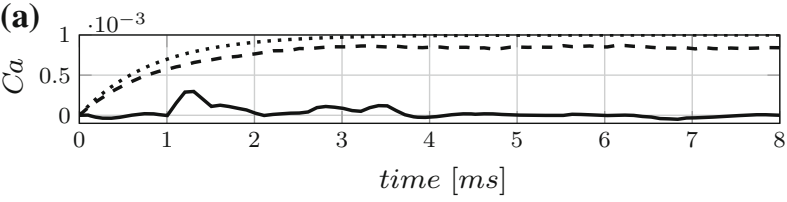

(b)

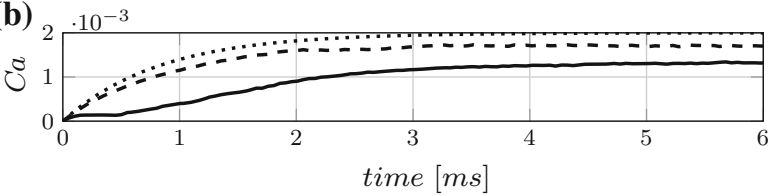

(c)
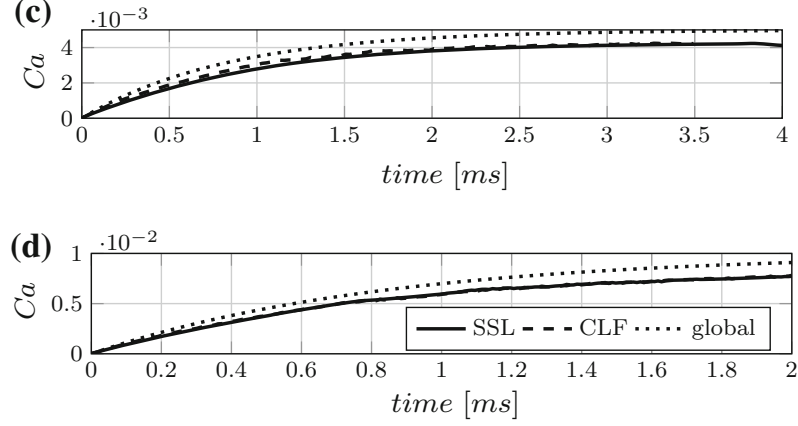

Fig. 14 Capillary numbers of imbibition processes versus time for different external pressure differences. a $\Delta p_{\mathrm{ex}}=-50 \mathrm{~Pa}, \mathbf{b} \Delta p_{\mathrm{ex}}=38 \mathrm{~Pa}, \mathbf{c} \Delta p_{\mathrm{ex}}=300 \mathrm{~Pa}$ and $\mathbf{d} \Delta p_{\mathrm{ex}}=736 \mathrm{~Pa}$

can write:

$$
\Delta p_{\mathrm{visc}}=\frac{12 \mu l v_{\mathrm{cl}}}{d^{2}}, \quad p_{\mathrm{c}}=\frac{2 \sigma_{\mathrm{wn}} \cos \theta_{0}}{d}
$$

In accordance with Washburn (1921), this leads to a constant wetting speed $v_{0}$ once steady flow $\left(\frac{\mathrm{d} v_{\mathrm{cl}}}{\mathrm{d} t}=0\right)$ is established. Then, Eq. (44) combined with Eq. 45 yields:

$$
v_{0}=\frac{\sigma_{\mathrm{wn}} d \cos \theta_{0}}{6 \mu l}+\frac{\Delta p_{\mathrm{ex}} d^{2}}{12 \mu l}
$$

According to Fig. 10, the transition zone of the principal dissipation source from fluid-solid interactions to viscous dissipation is in the range of $1 \times 10^{-3}<C a<1 \times 10^{-2}$, where we see a clear change in the slope of the curves. We performed four different simulations with the values of the external pressure difference $\Delta p_{\text {ex }}$ (see Table 4) chosen so that the steady flow velocity $v_{0}$ falls in that transition zone. In Fig. 14 , simulation results for the change in capillary number with time are presented. The results of the CLF model and the new stick-slip model are compared with the solution of the average momentum balance (Eq. 44). When fluid-solid interactions are negligible, i.e., large $\mathrm{Ca}$, the imbibition velocities calculated with CLF model should match the solution of Eq. (44). The slightly lower values obtained with the CLF model can be explained by the local rolling-type motion close to the interface (Dussan 1979). We also see that the simulation results of CLF model and SSL model are very similar for large Ca (Fig. 14c, d). For the case shown in Fig. 14b, stick-slip effect with unsteady $\mathrm{Ca}$ is significant. This is discussed qualitatively in Fig. 15 in "Appendix A". For an applied pressure difference 
of $\Delta p_{\mathrm{ex}}=-50 \mathrm{~Pa}$, a strong stick-slip behavior is apparent and the simulation results in very small $\mathrm{Ca}$ (almost no flow, see Fig. 14a). This big difference can be explained when the possible dynamic capillary pressures in the system are analyzed. In the simulations of the PET tape moving down into the liquids (see Sect. 4.1), the dynamic contact angle increased up to $\theta_{\mathrm{D}}=100^{\circ}$ for $C a=10^{-4}$. According to Eq. (45), this corresponds to a decrease in local capillary pressure from $p_{\mathrm{c}, 0}=203 \mathrm{~Pa}$ to $p_{\mathrm{c}, \mathrm{D}}=-253 \mathrm{~Pa}$ (note the negative value of $p_{\mathrm{c}, \mathrm{D}}$ ). Since at $C a=10^{-4}$, we are still below the transition zone, the wetting dynamic is much slower than the range that Washburn equation (Eq. 46) predicts.

\section{Concluding Remarks}

At low flow velocities in a two-phase system, fluid-solid interactions, which are not captured in the CLF model, account for most of the total energy dissipation. Therefore, an improved CLF model, which we call stick-slip (SSL) model, was developed in order to describe wetting processes which are mainly controlled by such fluid-solid interactions at the contact line. A major advantage of the stick-slip model, compared to models where the contact angles are included as boundary condition, is that the dynamic contact angles directly result from increased energy dissipation at the contact line. With the stick-slip model, strong reductions in the dynamics of wetting processes compared to Washburn's equation can be captured, as illustrated in Sect. 4.2. Comparisons of our simulation results with experimental data showed a good correlation for the dynamic contact angle with capillary numbers in the full range of wetting dynamics. Nevertheless, it should be stated that contact angle hysteresis in a static system, like in a liquid column within in a vertical tube (de Gennes et al. 2003), cannot be captured with our stick-slip model, since there is no additional dissipation without a moving contact line. To further increase accuracy in future SPH simulations, recent schemes with second order accuracy in time and space (Trask et al. 2015; Frontiere et al. 2017) could prove to be useful.

Acknowledgements This work was funded by the German Research Foundation (DFG) within the framework of the International Research Training Group "NUPUS, Non-linearities and upscaling in porous media" (IRTG 1398) and the research unit "Multiskalen-Analyse komplexer Dreiphasensysteme" (FOR 2397). The second author would like to thank European Research Council (ERC) for the support received under the ERC Advanced Grant Agreement No. 341225. The constructive comments of three anonymous reviewers helped to improve the manuscript significantly.

\section{Appendix A}

Dynamic contact angles $\theta_{\mathrm{D}}$ are usually modeled as a as a feature of viscous dissipation (de Gennes 1985). The term $\mu \Delta \mathbf{v}$ in the Navier-Stokes equation (Eq. 1) is the source of the viscous energy dissipation $\Phi$. Thus, the effect of stick-slip phenomena in our model can be expressed as follows. At a high capillary number ( $\mathrm{Ca}>0.1$ in the example given in Fig. 5), there is almost no viscosity increase and it becomes almost constant $\left(\mu_{\mathrm{SSL}} \approx \mu\right)$. Since $\Delta \mathbf{v}$ monotonically increases as the contact line velocity increases $\left(\frac{\mathrm{d} \Delta \mathbf{v}}{\mathrm{d} v_{\mathrm{cl}}}>0\right)$, the viscous dissipation is also increasing $\left(\frac{\mathrm{d} \Phi}{\mathrm{d} v_{\mathrm{cl}}}>0\right)$, because $\mu$ is almost constant. We refer to this behavior as steady contact line movement in Fig. 15. Now, with strongly decreasing viscosity and with increasing dynamics at slow wetting processes $\left(\frac{\mathrm{d} \mu_{\mathrm{SSL}}}{\mathrm{d} v_{\mathrm{cl}}}<0\right)$, it can happen that the 


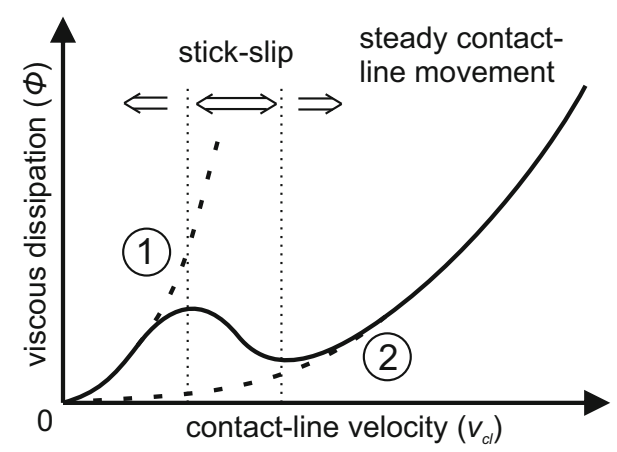

Fig. 15 Schematic plots of the viscous dissipation in the vicinity of a contact line in the transition between stick-slip wetting regime and a steady contact line movement. The dashed lines 1 and 2 represent the viscous dissipation with a constant high viscosity $\mu_{\mathrm{SSL}}=\mu(1+\alpha)$ (for $\mathrm{Ca} \rightarrow 0$ ) and constant low viscosity $\mu_{\mathrm{SSL}}=\mu$, respectively. The solid line is a qualitative plot when the new stick-slip model is applied

viscous dissipation decreases, while the contact line accelerates $\frac{\mathrm{d} \Phi}{\mathrm{d} v_{\mathrm{cl}}}<0$. This property of our model is in accordance with results from molecular-dynamics simulation of Thompson and Robbins (1990), where the frictional force is reduced in the transition from the ordered state, when the fluid molecules are sticking to the solid, to the unordered sliding state. Such a flow is unstable and will adjust itself toward one of the two possible stable states with $\frac{\mathrm{d} \Phi}{\mathrm{d} v_{\mathrm{cl}}}>0$. This stick-slip behavior is qualitatively shown in Fig. 15 .

\section{Appendix B}

An appropriate test case to analyze the accuracy of the applied SPH scheme is the twodimensional Taylor-Green flow. This test case is well discussed in the literature for the SPH method (Oger et al. 2016). We consider a Reynolds number $R e=\frac{l u_{\max }}{v}=100$ with characteristic length $l=1 \mathrm{~m}$, the maximum initial velocity $u_{\max }=1 \frac{\mathrm{m}}{\mathrm{s}}$, periodic boundary conditions and a resolution $l / \Delta x$ between 25 and 100. The initial divergence-free velocity field is given as:

$$
\begin{aligned}
& v_{x}^{*}=\sin \left(2 \pi x^{*}\right) \cos \left(2 \pi y^{*}\right) \\
& v_{y}^{*}=-\cos \left(2 \pi x^{*}\right) \sin \left(2 \pi y^{*}\right)
\end{aligned}
$$

The dimensionless velocities are $v_{x}^{*}=\frac{v_{x}}{v_{\max }}$ and $v_{y}^{*}=\frac{v_{y}}{v_{\max }}$, respectively. The dimensionless coordinates are $x^{*}=\frac{x}{l}$ and $y^{*}=\frac{y}{l}$, respectively. The analytic solution of the dimensionless kinetic energy of the system over time is given as:

$$
E_{\mathrm{kin}}^{*}=\frac{E_{\mathrm{kin}}}{E_{\mathrm{kin}}^{0}}=e^{\left(-16 \pi^{2} v t\right)}
$$

with the initial kinetic energy $E_{\text {kin }}^{0}$ and the kinematic viscosity $v$. The analytic solution for the local pressure field is given as:

$$
p^{*}(x, y)=\frac{1}{2}\left[\cos \left(4 \pi x^{*}\right)+\cos \left(4 \pi y^{*}\right)\right] e^{\left(-16 \pi^{2} / R e\right) t^{*}} .
$$


(a)

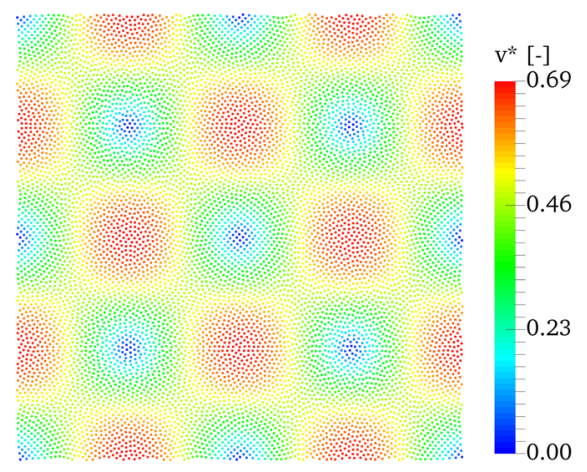

(b)

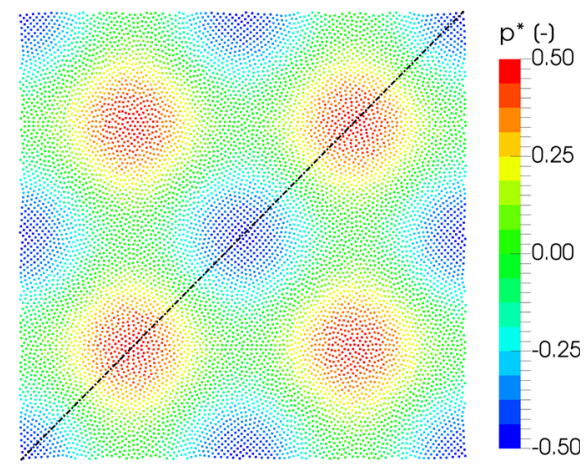

Fig. 16 Taylor-Green flow: Particle snapshots for $t^{*}=0.5$, a dimensionless velocity field $v^{*}, \mathbf{b}$ dimensionless pressure field $p^{*}$

(a)

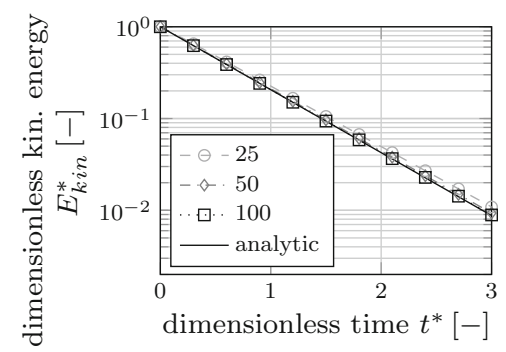

(b)

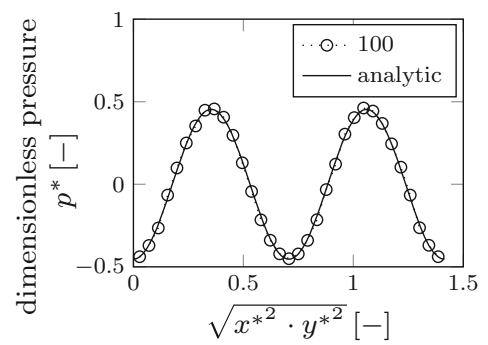

Fig. 17 Taylor-Green flow: convergence of the kinetic energy decay at $R e=100$ obtained with proposed ISPH scheme

In Fig. 16, we exemplary show the velocity and pressure distribution for $R e=100$ at $t^{*}=0.5$ with a resolution of $L / \Delta x=100$. In Fig. 17a, the convergence for the kinetic energy decay predicted by the proposed ISPH scheme is shown and compared against the analytic solution for $R e=100$ for resolutions $L / \Delta x=25, L / \Delta x=50$ and $L / \Delta x=100$. In Fig. 17b, the comparison between simulated dimensionless pressures $p^{*}$ and analytic solution is shown on the line between the points $P_{0}=(0 \mid 0)$ and $P_{1}=(1 \mid 1)$. The line is also plotted in Fig. $16 \mathrm{~b}$.

\section{References}

Adami, S., Hu, X.Y., Adams, N.A.: A new surface-tension formulation for multi-phase sph using a reproducing divergence approximation. J. Comput. Phys. 229(13), 5011-5021 (2010). https://doi.org/10.1016/j.jcp. 2010.03.022

Angeli, P., Gavriilidis, A.: Taylor flow in microchannels. In: Li, D. (ed.) Encyclopedia of Microfluidics and Nanofluidics, pp. 1971-1976. Springer, Boston (2008). https://doi.org/10.1007/978-0-387-48998-8_ 1526

Aslannejad, H., Hassanizadeh, S.M., Raoof, A., de Winter, D., Tomozeiu, N., van Genuchten, M.: Characterizing the hydraulic properties of a porous coating of paper using fib-sem tomography and $3 \mathrm{~d}$ pore-scale modeling. Chem. Eng. Sci. (2016). https://doi.org/10.1016/j.ces.2016.11.021

Blake, T.D.: The contact angle and two-phase flow. Ph.D. thesis, University of Bristol (1969) 
Blake, T.D.: Dynamic contact angles and wetting kinetics. In: Berg, J.C. (ed.) Wettability, Surfactant Science Series, pp. 251-310. M. Dekker, New York (1993)

Blake, T.D.: Forced wetting of a reactive surface. In: Interfaces, Wettability, Surface Forces and Applications: Special Issue in honour of the 65th Birthday of John Ralston, vol. 179-182, pp. 22-28 (2012). https:// doi.org/10.1016/j.cis.2012.06.002

Blake, T.D., Shikhmurzaev, Y.D.: Dynamic wetting by liquids of different viscosity. J. Colloid Interface Sci. 253(1), 196-202 (2002). https://doi.org/10.1006/jcis.2002.8513

Bonet, J., Lok, T.S.L.: Variational and momentum preservation aspects of smooth particle hydrodynamic formulations. Comput. Methods Appl. Mech. Eng. 180(1-2), 97-115 (1999). https://doi.org/10.1016/ S0045-7825(99)00051-1

Brackbill, J.U., Kothe, D.B., Zemach, C.: A continuum method for modeling surface tension. J. Comput. Phys. 100(2), 335-354 (1992). https://doi.org/10.1016/0021-9991(92)90240-Y

Brochard, F.: Motions of droplets on solid surfaces induced by chemical or thermal gradients. Langmuir 5(2), 432-438 (1989). https://doi.org/10.1021/la00086a025

Chen, Y., Kulenovic, R., Mertz, R.: Numerical study on the formation of taylor bubbles in capillary tubes. Nano Micro Mini Channels Comput. Heat Transf. 48(2), 234-242 (2009). https://doi.org/10.1016/j. ijthermalsci.2008.01.004

Cummins, S.J., Rudman, M.: An SPH projection method. J. Comput. Phys. 152(2), 584-607 (1999). https:// doi.org/10.1006/jcph.1999.6246

de Gennes, P.-G.: Wetting: statics and dynamics. Rev. Mod. Phys. 57(3), 827-863 (1985). https://doi.org/10. 1103/RevModPhys.57.827

de Gennes, P.-G., Brochard-Wyart, F., Quéré, D.: Capillarity and Wetting Phenomena: Drops, Bubbles, Pearls, Waves. Springer Science \& Business Media, Berlin (2003)

Dussan, E.B.: On the spreading of liquids on solid surfaces: static and dynamic contact lines. Annu. Rev. Fluid Mech. 11(1), 371-400 (1979)

Extrand, C.: Water contact angles and hysteresis of polyamide surfaces. J. Colloid Interface Sci. 248(1), 136-142 (2002)

Ferrari, A., Jimenez-Martinez, J., Le Borgne, T., Méheust, Y., Lunati, I.: Challenges in modeling unstable twophase flow experiments in porous micromodels. Water Resour. Res. 51(3), 1381-1400 (2015). https:// doi.org/10.1002/2014WR016384

Francois, M., Shyy, W.: Computations of drop dynamics with the immersed boundary method, part 2: drop impact and heat transfer. Numer. Heat Transf. B Fundam. 44(2), 119-143 (2003). https://doi.org/10. 1080/713836348

Frontiere, N., Raskin, C.D., Owen, J.M.: Crksph-a conservative reproducing kernel smoothed particle hydrodynamics scheme. J. Comput. Phys. 332((Supplement C)), 160-209 (2017). https://doi.org/10.1016/j. jcp.2016.12.004

Hao, L., Cheng, P.: Lattice boltzmann simulations of water transport in gas diffusion layer of a polymer electrolyte membrane fuel cell. J. Power Sources 195(12), 3870-3881 (2010). https://doi.org/10.1016/j. jpowsour.2009.11.125

Hassanizadeh, S.M., Gray, W.G.: Thermodynamic basis of capillary pressure in porous media. Water Resour. Res. 29(10), 3389-3405 (1993). https://doi.org/10.1029/93WR01495

Hu, X.Y., Adams, N.A.: A multi-phase SPH method for macroscopic and mesoscopic flows. J. Comput. Phys. 213(2), 844-861 (2006). https://doi.org/10.1016/j.jcp.2005.09.001

Hu, X.Y., Adams, N.A.: An incompressible multi-phase SPH method. J. Comput. Phys. 227(1), 264-278 (2007). https://doi.org/10.1016/j.jcp.2007.07.013

Huber, M., Dobesch, D., Kunz, P., Hirschler, M., Nieken, U.: Influence of orifice type and wetting properties on bubble formation at bubble column reactors. Chem. Eng. Sci. 152, 151-162 (2016a). https://doi.org/ 10.1016/j.ces.2016.06.002

Huber, M., Keller, F., Säckel, W., Hirschler, M., Kunz, P., Hassanizadeh, S.M., Nieken, U.: On the physically based modeling of surface tension and moving contact lines with dynamic contact angles on the continuum scale. J. Comput. Phys. 310, 459-477 (2016b). https://doi.org/10.1016/j.jcp.2016.01.030

Joekar, N.V., Hassanizadeh, S.M., Pyrak-Nolte, L.J., Berentsen, C.: Simulating drainage and imbibition experiments in a high-porosity micromodel using an unstructured pore network model. Water Resour. Res. 45(2), W02,430 (2009). https://doi.org/10.1029/2007WR006641

Johnson, R.E., Dettre, R.H.: Contact angle hysteresis. III. Study of an idealized heterogeneous surface. J. Phys. Chem. 68(7), 1744-1750 (1964). https://doi.org/10.1021/j100789a012

Kunz, P., Hirschler, M., Huber, M., Nieken, U.: Inflow/outflow with dirichlet boundary conditions for pressure in ISPH. J. Comput. Phys. 326, 171-187 (2016a). https://doi.org/10.1016/j.jcp.2016.08.046 
Kunz, P., Zarikos, I.M., Karadimitriou, N.K., Huber, M., Nieken, U., Hassanizadeh, S.M.: Study of multi-phase flow in porous media: comparison of SPH simulations with micro-model experiments. Transp. Porous Media 114(2), 581-600 (2016b). https://doi.org/10.1007/s11242-015-0599-1

Lafaurie, B., Nardone, C., Scardovelli, R., Zaleski, S., Zanetti, G.: Modelling merging and fragmentation in multiphase flows with surfer. J. Comput. Phys. 113(1), 134-147 (1994). https://doi.org/10.1006/jcph. 1994.1123

Monaghan, J.J.: Smoothed particle hydrodynamics. Rep. Prog. Phys. 68(8), 1703 (2005). http://stacks.iop. org/0034-4885/68/i=8/a=R01

Monaghan, J.J.: Smoothed particle hydrodynamics and its diverse applications. Annu. Rev. Fluid Mech. 44(1), 323-346 (2011). https://doi.org/10.1146/annurev-fluid-120710-101220

Morris, J.P., Fox, P.J., Zhu, Y.: Modeling low reynolds number incompressible flows using SPH. J. Comput. Phys. 136(1), 214-226 (1997). https://doi.org/10.1006/jcph.1997.5776

Oger, G., Marrone, S., Le Touzé, D., de Leffe, M.: SPH accuracy improvement through the combination of a quasi-Lagrangian shifting transport velocity and consistent ALE formalisms. J. Comput. Phys. 313(Supplement C), 76-98 (2016). https://doi.org/10.1016/j.jcp.2016.02.039

Renardy, M., Renardy, Y., Li, J.: Numerical simulation of moving contact line problems using a volume-of-fluid method. J. Comput. Phys. 171(1), 243-263 (2001). https://doi.org/10.1006/jcph.2001.6785

Sheng, P., Zhou, M.: Immiscible-fluid displacement: contact-line dynamics and the velocity-dependent capillary pressure. Phys. Rev. A 45(8), 5694-5708 (1992)

Shikhmurzaev, Y.D.: Moving contact lines in liquid/liquid/solid systems. J. Fluid Mech. 334, 211-249 (1997). https://doi.org/10.1017/S0022112096004569

Sussman, M., Fatemi, E., Smereka, P., Osher, S.: An improved level set method for incompressible two-phase flows. Comput. Fluids 27(5-6), 663-680 (1998). https://doi.org/10.1016/S0045-7930(97)00053-4

Szewc, K., Pozorski, J., Minier, J.P.: Analysis of the incompressibility constraint in the smoothed particle hydrodynamics method. Int. J. Numer. Methods Eng. 92(4), 343-369 (2012). https://doi.org/10.1002/ nme.4339

Tartakovsky, A., Meakin, P.: Modeling of surface tension and contact angles with smoothed particle hydrodynamics. Phys. Rev. E 72(2), 26,301 (2005). https://doi.org/10.1103/PhysRevE.72.026301

Thompson, P.A., Robbins, M.O.: Simulations of contact-line motion: slip and the dynamic contact angle. Phys. Rev. Lett. 63(7), 766-769 (1989)

Thompson, P.A., Robbins, M.O.: Origin of stick-slip motion in boundary lubrication. Science 250(4982), 792-794 (1990). https://doi.org/10.1126/science.250.4982.792

Trask, N., Maxey, M., Kim, K., Perego, M., Parks, M.L., Yang, K., Xu, J.: A scalable consistent second-order SPH solver for unsteady low Reynolds number flows. Comput. Methods Appl. Mech. Eng. 289, 155-178 (2015). https://doi.org/10.1016/j.cma.2014.12.027

Wang, J., Do-Quang, M., Cannon, J.J., Yue, F., Suzuki, Y., Amberg, G., Shiomi, J.: Surface structure determines dynamic wetting. Sci. Rep. 5, 8474 EP (2015)

Washburn, E.W.: The dynamics of capillary flow. Phys. Rev. 17(3), 273-283 (1921). https://doi.org/10.1103/ PhysRev.17.273

Wendland, H.: Piecewise polynomial, positive definite and compactly supported radial functions of minimal degree. Adv. Comput. Math. 4(1), 389-396 (1995). https://doi.org/10.1007/BF02123482 